\title{
Erik Beek and Rick R. van Rijn (Eds): Diagnostic Pediatric Ultrasound
}

\author{
Georg Thieme Verlag KG, 2016. ISBN: 978-3-13169731-8
}

\author{
Camilla Russo $^{1} \cdot$ Gianfranco Vallone $^{1}$
}

Published online: 11 June 2016

(C) Springer-Verlag Berlin Heidelberg 2016

This is an interesting book designed to underline the crucial role of ultrasonography in paediatric imaging. The editors are two prominent Dutch authorities in the field, Erik Beek (Consulting Radiologist at the University Medical Center, Utrecht) and Rick R. van Rijn (Professor at the Emma Children's Hospital, Academic Medical Center, Amsterdam), and the contributors include many European experts in paediatric diagnostic imaging.

The book consists of 660 pages in 18 chapters. The first chapter is dedicated to the importance of correct child examination and a child-friendly environment in a hospital ward, while the second chapter provides a concise overview of the basic principles of ultrasonography. In the following 15 chapters the approach to paediatric pathology is by single organs and systems, starting from neonatal ultrasonography. Before considering pathology, the authors always provide a description of embryology, normal anatomy and the most common anatomical variants. The last chapter focuses on a brief overview of the most common ultrasound-guided interventional procedures used by paediatric radiologists.

The volume contains 2,025 illustrations, including diagnostic images, diagrammatic representations and panels illustrated with pictures, that make the correct interpretation of the imaging easier for both radiologists and nonradiologists. In addition, many chapters are accompanied by $\mathrm{CD}$ video material that enriches the information contained in the book; additional teaching files and videos on ultrasonography are available from the website MediaCenter.thieme.com.

It is immediately clear that the main goal of this text is to emphasize the centrality of sonography in paediatric diagnostic

Gianfranco Vallone

gianfranco.vallone2@unina.it

1 Università degli Studi di Napoli “Federico II”, Naples, Italy imaging in daily practice. It explains in detail why ultrasonography must always be the first-line imaging modality in the approach to paediatric patients because it offers a safe and noninvasive method for obtaining data. According to the aim of the authors, the discussion leaves little room for consideration of other diagnostic methods, such as CT and MRI. Thus, it offers a wide range of information about ultrasonography, especially for the first time for paediatricians and paediatric surgeons, but provides only a partial view of paediatric diagnostic imaging for radiology specialists. However, this aspect probably goes beyond the intentions of the volume and such supplementary information can readily be obtained from other texts.

The main strength of this book is the huge variety of highquality images used, particularly normal reference images that complement and provide a means of comparison with pathological findings, which make the text easier to understand and consult. Moreover, for less expert operators, the video content offers a specific reference model to use in daily practice. The only deficiency of the text is attributable to the brief and incomplete description of ultrasound-guided interventional procedures, that are not discussed in sufficient detail.

In conclusion, the book can be considered a valid tool for specialists such as radiologists, paediatricians and paediatric surgeons. On the one hand, it is necessary to appreciate the intention to combine clinical evaluation and ultrasonography protocols to optimize diagnostic performance, by adapting technical methods of examination to the individual patient. On the other hand, for a complete development of the argument, it would have been desirable to complete the final part of the text by adding a more detailed description of ultrasound-guided interventional procedures, that are increasingly common in clinical practice.

Overall, the book is clear, user-friendly and easy to read; with a large number of didactic tables and images; it provides a complete compendium of the key points of diagnostic paediatric ultrasonography. 